\title{
On the Number of Limit Cycles of a Piecewise Quadratic Near-Hamiltonian System
}

\author{
Jing Tian \\ Department of Mathematics, Shanghai Normal University, Shanghai 200234, China \\ Correspondence should be addressed to Jing Tian; tj0634@163.com
}

Received 24 June 2014; Accepted 23 July 2014; Published 14 October 2014

Academic Editor: Tonghua Zhang

Copyright (C) 2014 Jing Tian. This is an open access article distributed under the Creative Commons Attribution License, which permits unrestricted use, distribution, and reproduction in any medium, provided the original work is properly cited.

This paper is concerned with the problem for the maximal number of limit cycles for a quadratic piecewise near-Hamiltonian system. By using the method of the first order Melnikov function, we find that it can have 8 limit cycles.

\section{Introduction and Main Results}

Recently, piecewise smooth systems attracted many researchers' attention since many real processes and different modern devices can be modeled by them; see $[1,2]$ and references therein. Due to their nonsmoothness, these systems can have richer dynamical phenomena than the smooth ones (see [3-5] and the references cited therein). For instance, nonsmooth system can have the sliding phenomena and in $[6,7]$ Giannakopoulos and Pliete have studied the existence of sliding cycles and sliding homoclinic cycles for a planar relay control feedback systems.

As we have seen, studying the existence and number of limit cycles is one of the main problems for piecewise smooth systems; see [8-16]. Limit cycles of piecewise smooth linear differential systems defined on two half-planes separated by a straight line $x=0$ or $y=0$ have been studied recently in [8-11], from which one can find that 3 limit cycles can appear for piecewise smooth linear systems. From [12-14], we can know that piecewise linear and quadratic near-Hamiltonian systems can have 2 and 3 limit cycles, respectively. In fact, these papers investigated the problem for limit cycle bifurcations of piecewise linear Hamiltonian systems under piecewise polynomial perturbations of degree $n$, obtaining some new and interesting results. Furthermore, the authors of [16] studied a piecewise quadratic Hamiltonian system (one side linear and another side quadratic) perturbed inside the class of piecewise polynomial differential systems of degree $n$ and achieved that it can have $2 n+[(n+1) / 2]$ limit cycles, which implies that piecewise quadratic nearHamiltonian systems can have 5 limit cycles. The authors of [15] also obtained this result by perturbed quadratic polynomial differential systems containing an isochronous center with piecewise quadratic polynomials of degree 2 .

In this paper, we mainly study the problem for the maximal number of a quadratic near-Hamiltonian system. That is to say, we consider a system of the form

$$
\dot{x}=y+\varepsilon p(x, y), \quad \dot{y}=-g(x)+\varepsilon q(x, y),
$$

where $\varepsilon>0$ is a small parameter,

$$
g(x)= \begin{cases}a_{2} x^{2}+a_{1} x+a_{0}, & x<0, \\ b_{2} x^{2}+b_{1} x+b_{0}, & x>0\end{cases}
$$

with $a_{2} b_{2} \neq 0$, and

$$
\begin{aligned}
& p(x, y)= \begin{cases}\sum_{i+j=0}^{2} a_{i j}^{-} x^{i} y^{j}, & x<0, \\
\sum_{i+j=0}^{2} a_{i j}^{+} x^{i} y^{j}, & x>0,\end{cases} \\
& q(x, y)= \begin{cases}\sum_{i+j=0}^{2} b_{i j}^{-} x^{i} y^{j}, & x<0, \\
\sum_{i+j=0}^{2} b_{i j}^{+} x^{i} y^{j}, & x>0 .\end{cases}
\end{aligned}
$$

Then, our main results can be stated as follows. 


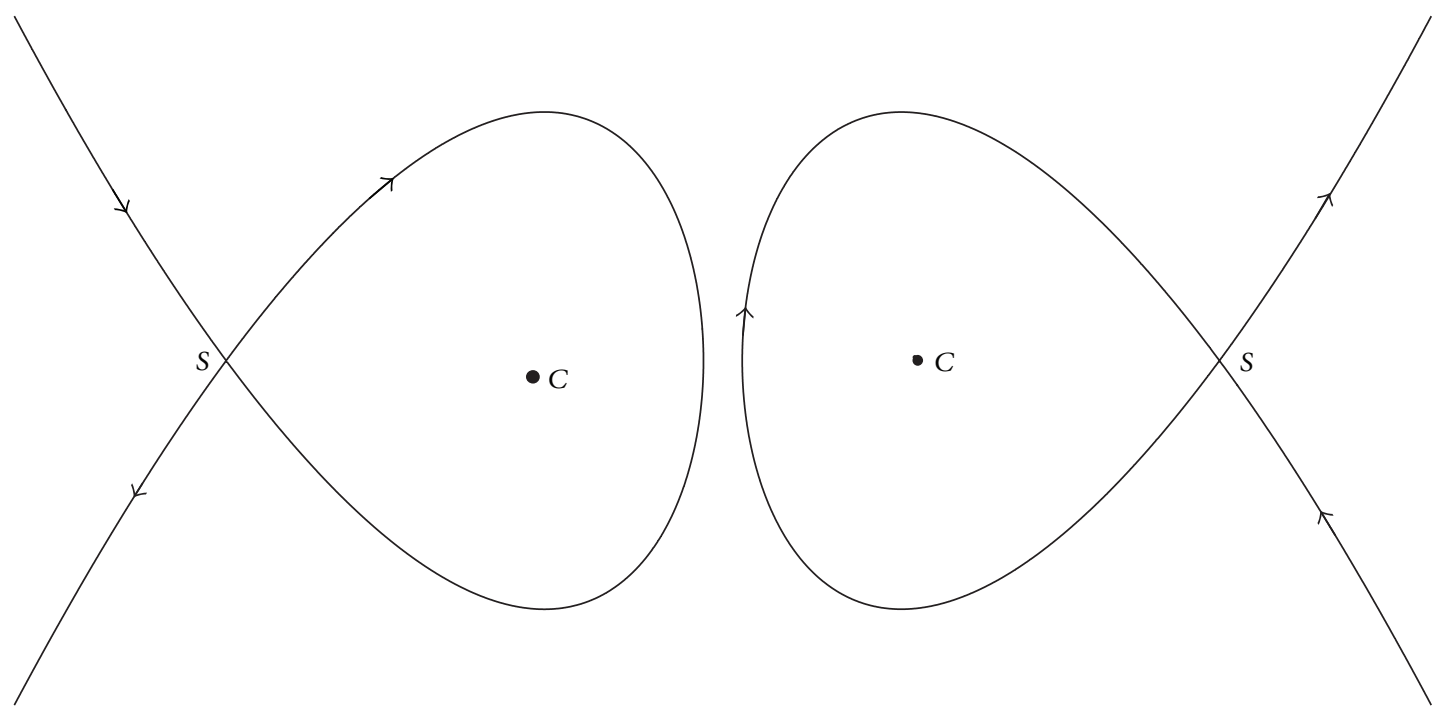

(a) $a<0$

(b) $a>0$

FIgURE 1: Level curves of $(1 / 2) y^{2}+(1 / 3) a x^{3}+(1 / 2) b x^{2}+c x=h$.

Theorem 1. There exists a system of form (1) which has 8 limit cycles.

In the next section, the proof of Theorem 1 is presented.

\section{Proof of Theorem 1}

In this section, we divide the proof into two subsections: one section is to present some preliminary knowledge, which is useful to verify our main result; another section is to give the process of the proof of Theorem 1 .

2.1. Preliminaries. Clearly, system (1) has the following two subsystems:

$$
\begin{aligned}
& \dot{x}=y+\varepsilon \sum_{i+j=0}^{2} a_{i j}^{-} x^{i} y^{j}, \\
& \dot{y}=-\left(a_{2} x^{2}+a_{1} x+a_{0}\right)+\varepsilon \sum_{i+j=0}^{2} b_{i j}^{-} x^{i} y^{j}, \\
& \dot{x}=y+\varepsilon \sum_{i+j=0}^{2} a_{i j}^{+} x^{i} y^{j}, \\
& \dot{y}=-\left(b_{2} x^{2}+b_{1} x+b_{0}\right)+\varepsilon \sum_{i+j=0}^{2} b_{i j}^{+} x^{i} y^{j},
\end{aligned}
$$

which are called left and right subsystems, respectively. And systems $\left.(4 \mathrm{a})\right|_{\varepsilon=0}$ and $\left.(4 \mathrm{~b})\right|_{\varepsilon=0}$ are Hamiltonian with Hamiltonian functions, respectively,

$$
\begin{aligned}
& H^{-}(x, y)=\frac{1}{2} y^{2}+\frac{1}{3} a_{2} x^{3}+\frac{1}{2} a_{1} x^{2}+a_{0} x, \\
& H^{+}(x, y)=\frac{1}{2} y^{2}+\frac{1}{3} b_{2} x^{3}+\frac{1}{2} b_{1} x^{2}+b_{0} x .
\end{aligned}
$$

For systems $\left.(4 a)\right|_{\varepsilon=0}$ and $\left.(4 b)\right|_{\varepsilon=0}$, we make the following assumption:

(H) $a_{1}^{2}>4 a_{2} a_{0}, b_{1}^{2}>4 b_{2} b_{0}$.

Then, under the condition $(\mathrm{H})$, for $\varepsilon=0$, system (4a) (resp., (4b)) has a hyperbolic saddle $S_{1}=\left(\alpha_{S_{1}}, 0\right)$ (resp., $\left.S_{2}=\left(\alpha_{S_{2}}, 0\right)\right)$ and an elementary center $C_{1}=\left(\alpha_{C_{1}}, 0\right)$ (resp., $\left.C_{2}=\left(\alpha_{C_{2}}, 0\right)\right)$, where

$$
\begin{array}{ll}
\alpha_{S_{1}}=\frac{-a_{1}-\sqrt{a_{1}^{2}-4 a_{2} a_{0}}}{2 a_{2}}, & \alpha_{S_{2}}=\frac{-b_{1}-\sqrt{b_{1}^{2}-4 b_{2} b_{0}}}{2 b_{2}}, \\
\alpha_{C_{1}}=\frac{-a_{1}+\sqrt{a_{1}^{2}-4 a_{2} a_{0}}}{2 a_{2}}, & \alpha_{C_{2}}=\frac{-b_{1}+\sqrt{b_{1}^{2}-4 b_{2} b_{0}}}{2 b_{2}} .
\end{array}
$$

We will assume that

$$
a_{2}<0 \text {, }
$$

since, for $a_{2}>0$, we can make a variable transformation $x \rightarrow$ $-x, y \rightarrow y$, together with a time rescaling $t \rightarrow-t$ such that the resulting system satisfies (7). Further, note that there exist 2 possible cases for level curves of $H(x, y)=(1 / 2) y^{2}+$ $(1 / 3) a x^{3}+(1 / 2) b x^{2}+c x=h$ on the plane, where $a \neq 0$, $b^{2}>4 a c$; see Figure 1 .

We remark that, in Figure $1, C=\left(\left(-b+\sqrt{b^{2}-4 a c}\right) / 2 a, 0\right)$, $S=\left(\left(-b-\sqrt{b^{2}-4 a c}\right) / 2 a, 0\right)$. Then, one can obtain 9 possible phase portraits of system (1) $\left.\right|_{\varepsilon=0}$ under $(\mathrm{H})$ and (7) when it has a generalized closed orbit and two hyperbolic saddles; see Figure 2. For system (1) $\left.\right|_{\varepsilon=0}$, a generalized closed orbit passes through the $y$-axis two times.

Now, we study limit cycle bifurcations on system (1). For simplicity, we consider the case

$$
\alpha_{S_{1}}<0<\alpha_{S_{2}}<\alpha_{C_{2}}, \quad H^{-}\left(S_{1}\right)=H^{+}\left(S_{2}\right) \triangleq h_{1} ;
$$




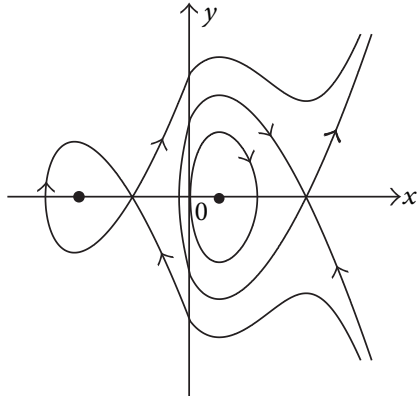

(a) $\alpha_{S_{1}}<0<\alpha_{C_{2}}<\alpha_{S_{2}}, H^{-}\left(S_{1}\right)>$ $H^{+}\left(S_{2}\right)$

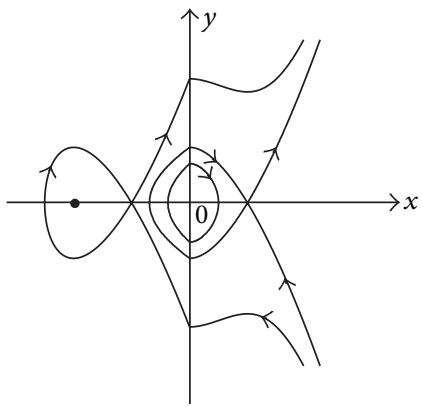

(d) $\alpha_{S_{1}}<0, \alpha_{C_{2}} \leq 0<\alpha_{S_{2}}, H^{-}\left(S_{1}\right)>$ $H^{+}\left(S_{2}\right)$

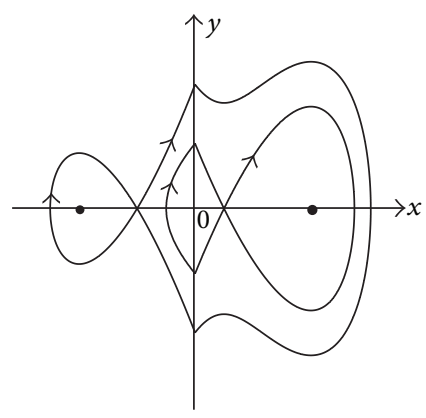

(g) $\alpha_{S_{1}}<0<\alpha_{S_{2}}<\alpha_{C_{2}}, H^{-}\left(S_{1}\right)>$ $H^{+}\left(S_{2}\right)$

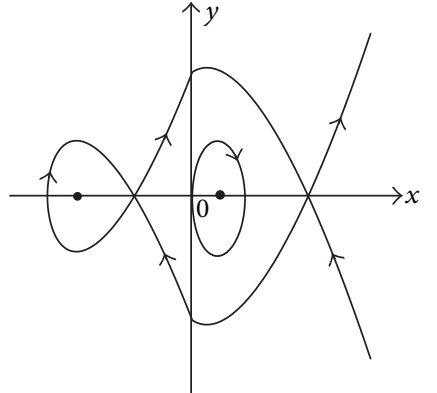

(b) $\alpha_{S_{1}}<0<\alpha_{C_{2}}<\alpha_{S_{2}}, H^{-}\left(S_{1}\right)=$ $H^{+}\left(S_{2}\right)$

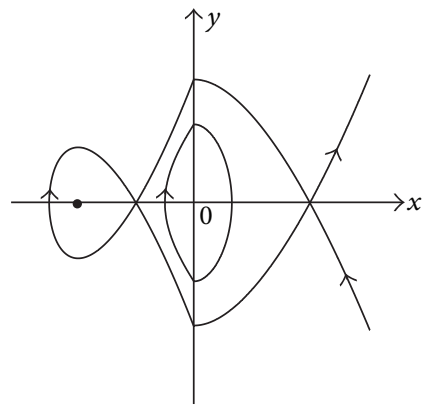

(e) $\alpha_{S_{1}}<0, \alpha_{C_{2}} \leq 0<\alpha_{S_{2}}, H^{-}\left(S_{1}\right)=$ $H^{+}\left(S_{2}\right)$

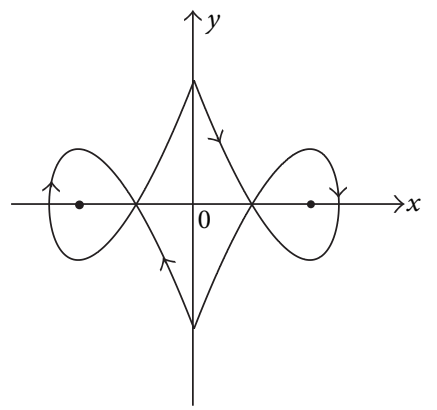

$\begin{aligned} \text { (h) } \alpha_{S_{1}}<0<\alpha_{S_{2}}<\alpha_{C_{2}}, H^{-}\left(S_{1}\right)= & \text { (i) } \alpha_{S_{1}}<0<\alpha_{S_{2}}<\alpha_{C_{2}}, H^{\top}\left(S_{1}\right)< \\ & H^{+}\left(S_{2}\right)\end{aligned}$

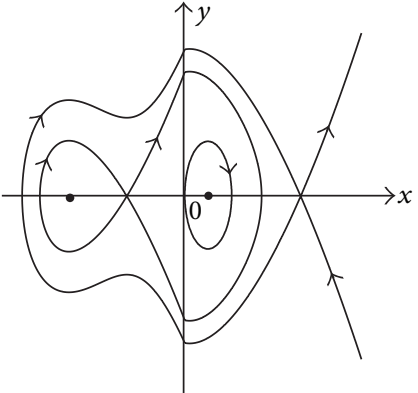

(c) $\alpha_{S_{1}}<0<\alpha_{C_{2}}<\alpha_{S_{2}}, H^{-}\left(S_{1}\right)<$ $H^{+}\left(S_{2}\right)$

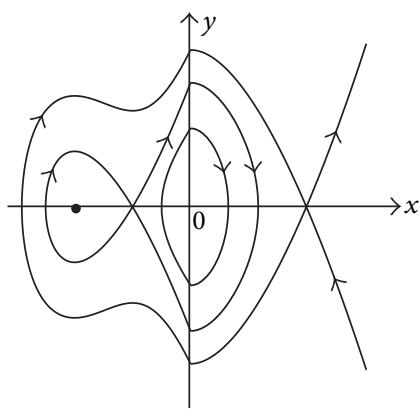

(f) $\alpha_{S_{1}}<0, \alpha_{C_{2}} \leq 0<\alpha_{S_{2}}, H^{-}\left(S_{1}\right)<$ $H^{+}\left(S_{2}\right)$

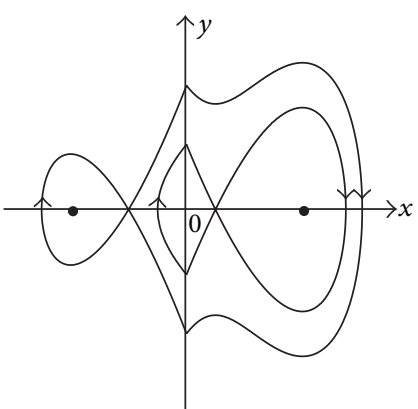

Figure 2: Possible portraits of (1) $\left.\right|_{\varepsilon=0}$ with two saddles and a generalized closed orbit.

that is,

$$
\begin{gathered}
a_{1}^{2}>4 a_{2} a_{0}, \quad b_{1}^{2}>4 b_{2} b_{0}, \quad a_{2}<0, \quad b_{2}>0, \\
a_{1}<0, \quad b_{1}<0, \quad a_{0}<0, \quad b_{0}>0, \\
\frac{a_{1}^{3}-6 a_{0} a_{1} a_{2}+\left(a_{1}^{2}-4 a_{0} a_{2}\right)^{3 / 2}}{a_{2}^{2}} \\
=\frac{b_{1}^{3}-6 b_{0} b_{1} b_{2}+\left(b_{1}^{2}-4 b_{0} b_{2}\right)^{3 / 2}}{b_{2}^{2}} .
\end{gathered}
$$

Then, the phase portrait of system (1) $\left.\right|_{\mathcal{E}=0}$ is Figure $2(\mathrm{~h})$, and there are four families of periodic orbits given by

$L_{1 h}=\left\{(x, y) \mid H^{-}(x, y)=h, x_{0}^{-}<x<\alpha_{S_{1}}\right\}, \quad h_{0}^{-}<h<h_{1}$, $L_{2 h}=\left\{(x, y) \mid H^{+}(x, y)=h, \alpha_{S_{2}}<x<x_{0}^{+}\right\}, \quad h_{0}^{+}<h<h_{1}$,

$$
L_{3 h}=L_{3 h}^{-} \cup L_{3 h}^{+}=\left\{(x, y) \mid H^{-}(x, y)=h, \alpha_{S_{1}}<x<0\right\}
$$$$
\cup\left\{(x, y) \mid H^{+}(x, y)=h, 0<x<\alpha_{S_{2}}\right\},
$$$$
0<h<h_{1}
$$$$
L_{h}=L_{h}^{-} \cup L_{h}^{+}=\left\{(x, y) \mid H^{-}(x, y)=h, x<0\right\}
$$

$$
\cup\left\{(x, y) \mid H^{+}(x, y)=h, x>0\right\}, \quad h>h_{1},
$$

where $H^{-}\left(x_{0}^{-}, 0\right)=h_{1}, H^{+}\left(x_{0}^{+}, 0\right)=h_{1}$ and $H^{-}\left(C_{1}\right)=h_{0}^{-}$, $H^{+}\left(C_{2}\right)=h_{0}^{+}$. Further, we have

$\lim _{h \rightarrow h_{1}} L_{1 h}=L_{1}, \quad \lim _{h \rightarrow h_{0}^{-}} L_{1 h}=C_{1}, \quad \lim _{h \rightarrow h_{1}} L_{2 h}=L_{2}$,

$\lim _{h \rightarrow h_{0}^{+}} L_{1 h}=C_{2}, \quad \lim _{h \rightarrow h_{1}} L_{3 h}^{-}=L^{-}$, 


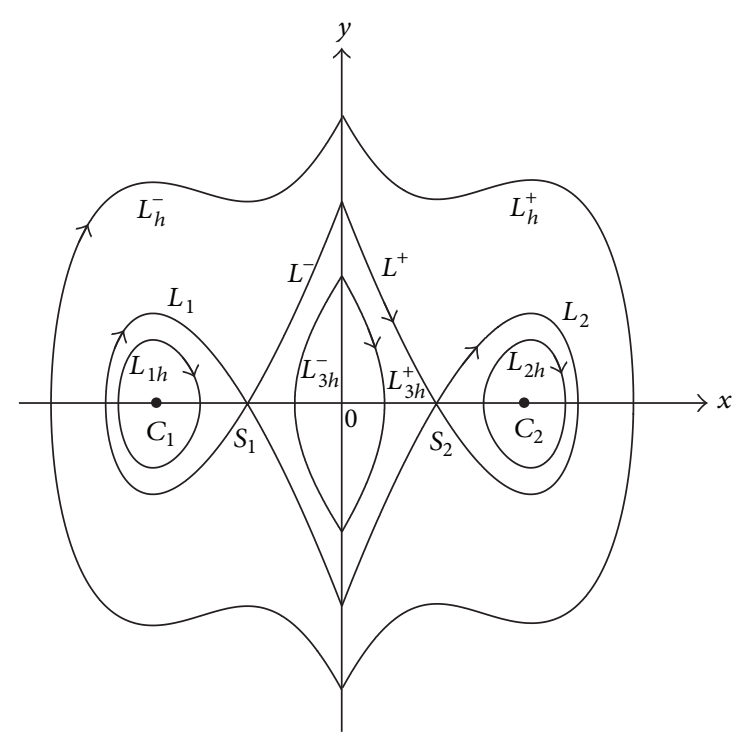

Figure 3: Phase portrait of system (1) $\left.\right|_{\varepsilon=0}$ under (9).

$$
\begin{aligned}
& \lim _{h \rightarrow h_{1}} L_{3 h}^{+}=L^{+}, \quad \lim _{h \rightarrow 0} L_{3 h}^{-}=\lim _{h \rightarrow 0} L_{3 h}^{+}=(0,0), \\
& \lim _{h \rightarrow h_{1}} L_{h}^{-}=L_{1} \cup L^{-}, \quad \lim _{h \rightarrow h_{1}} L_{h}^{+}=L_{2} \cup L^{+} ;
\end{aligned}
$$

see Figure 3. From (11), one can know that, as $h$ approaches $h_{1}$, the limit of $L_{h}$ is $L_{1} \cup L^{-} \cup L_{2} \cup L^{+}$denoted by $L$, which is a generalized polycycle with two hyperbolic saddles.

Then, from Theorem 1.1 of [17], for system (1), we have four first order Melnikov functions as follows:

$$
\begin{aligned}
M_{1}(h)= & \oint_{L_{1 h+j=0}} \sum_{i j}^{2} b_{i j}^{-} x^{i} y^{j} d x-\sum_{i+j=0}^{2} a_{i j}^{-} x^{i} y^{j} d y, \\
& h\left(h_{0}^{-}, h_{1}\right), \\
M_{2}(h)= & \oint_{L_{2 h}} \sum_{i+j=0}^{2} b_{i j}^{+} x^{i} y^{j} d x-\sum_{i+j=0}^{2} a_{i j}^{+} x^{i} y^{j} d y, \\
M_{3}(h)= & M_{3}^{-}(h)+M_{3}^{+}(h)=\int_{L_{3 h}^{-}} \sum_{i+j=0}^{2} b_{i j}^{-} x^{i} y^{j} d x \\
& -\sum_{i+j=0}^{2} a_{i j}^{-} x^{i} y^{j} d y+\int_{L_{3 h}^{+}} \sum_{i+j=0}^{2} b_{i j}^{+} x^{i} y^{j} d x \\
& -\sum_{i+j=0}^{2} a_{i j}^{+} x^{i} y^{j} d y, \quad h \in\left(0, h_{1}\right),
\end{aligned}
$$

$$
\begin{aligned}
M(h)= & M^{-}(h)+M^{+}(h)=\int_{L_{h}^{-}} \sum_{i+j=0}^{2} b_{i j}^{-} x^{i} y^{j} d x \\
& -\sum_{i+j=0}^{2} a_{i j}^{-} x^{i} y^{j} d y+\int_{L_{h}^{+}} \sum_{i+j=0}^{2} b_{i j}^{+} x^{i} y^{j} d x \\
& -\sum_{i+j=0}^{2} a_{i j}^{+} x^{i} y^{j} d y, \quad h \in\left(h_{1},+\infty\right),
\end{aligned}
$$

where $L_{1 h}, L_{2 h}, L_{3 h}^{-}, L_{3 h}^{+}, L_{h}^{-}$, and $L_{h}^{+}$are given in (10).

Now, we deduce the expansion of $M_{i}(h), i=1,2,3$, and $M(h)$ near $h=h_{1}$ in the above. First, we have the following.

Lemma 2. The function $M_{3}^{-}(h)$ in (12) has the following expression:

$$
\begin{aligned}
M_{3}^{-}(h)= & c_{0}+c_{1}\left(h-h_{1}\right) \ln \left(h_{1}-h\right)+c_{2}\left(h-h_{1}\right) \\
+c_{3}\left(h-h_{1}\right)^{2} \ln \left(h_{1}-h\right)+ & O\left(\left(h-h_{1}\right)^{2}\right), \\
0 & <h_{1}-h \ll 1,
\end{aligned}
$$

where

$$
\begin{aligned}
& c_{0}=\int_{L^{-}} {\left[\left(a_{10}^{-}+b_{01}^{-}\right) y+\left(b_{11}^{-}+2 a_{20}^{-}\right) x y\right.} \\
&\left.+\frac{1}{2}\left(a_{11}^{-}+2 b_{02}^{-}\right) y^{2}\right] d x-2 a_{00}^{-} \sqrt{2 h_{1}} \\
&-\frac{4}{3} h_{1} \sqrt{2 h_{1} a_{02}^{-}}, \\
& c_{1}=-\frac{1}{\left(a_{1}^{2}-4 a_{0} a_{2}\right)^{1 / 4}}\left[\left(a_{10}^{-}+b_{01}^{-}\right)+\alpha_{S_{1}}\left(2 a_{20}^{-}+b_{11}^{-}\right)\right], \\
&\left.c_{2}\right|_{c_{1}=0}=\int_{L^{-}}\left[\left(a_{10}^{-}+b_{01}^{-}\right)+\left(b_{11}^{-}+2 a_{20}^{-}\right) x+\left(a_{11}^{-}+2 b_{02}^{-}\right) y\right] d t \\
& c_{3}=\frac{\sqrt{2 h_{1}}}{2\left(a_{1}^{2}-\right.} a_{00}^{-}-2 \sqrt{2 h_{1}} a_{02}^{-}, \\
&\left.a_{0} a_{2}\right)^{5 / 4}
\end{aligned}
$$

Proof. Denote by $U$ a disk of diameter $\epsilon_{0}>0$ with its center at $S_{1}$. Then, for $\epsilon_{0}>0$ small, rewrite $M_{3}^{-}(h)=I_{1}(h)+I_{2}(h)$, where

$$
\begin{aligned}
& I_{1}(h)=\int_{\bar{L}_{3 h}^{-}} \sum_{i+j=0}^{2} b_{i j}^{-} x^{i} y^{j} d x-\sum_{i+j=0}^{2} a_{i j}^{-} x^{i} y^{j} d y, \\
& I_{2}(h)=\int_{\tilde{L}_{3 h}^{-}} \sum_{i+j=0}^{2} b_{i j}^{-} x^{i} y^{j} d x-\sum_{i+j=0}^{2} a_{i j}^{-} x^{i} y^{j} d y
\end{aligned}
$$

with

$$
\bar{L}_{3 h}^{-}=L_{3 h}^{-} \cap U, \quad \widetilde{L}_{3 h}^{-}=L_{3 h}^{-} \backslash \overline{L_{3 h}} .
$$


Note that $I_{2}(h)$ is a $C^{\infty}$ function for $h_{1}-h>0$ small. Thus, it suffices to investigate the expansion of $I_{1}(h)$ for $0<h_{1}-h \ll$ 1. To do this, we make a variable transformation

$$
\begin{gathered}
u=\left(a_{1}^{2}-4 a_{0} a_{2}\right)^{1 / 4}\left(x-\alpha_{S_{1}}\right), \quad v=y, \\
\tau=\left(a_{1}^{2}-4 a_{0} a_{2}\right)^{1 / 4} t .
\end{gathered}
$$

Then system (4a) becomes

$$
\begin{aligned}
& \dot{u}=v+\varepsilon \widetilde{p}^{-}(u, v), \\
& \dot{v}=u-\frac{a_{2} u^{2}}{\left(a_{1}^{2}-4 a_{0} a_{2}\right)^{3 / 4}}+\varepsilon \widetilde{q}^{-}(u, v),
\end{aligned}
$$

where

$$
\begin{aligned}
\widetilde{p}^{-}(u, v) & =\sum_{i+j=0}^{2} \widetilde{a}_{i j}^{-} u^{i} v^{j}=\sum_{i+j=0}^{2} a_{i j}^{-}\left[\frac{u}{\left(a_{1}^{2}-4 a_{0} a_{2}\right)^{1 / 4}}+\alpha_{S_{1}}\right]^{i} v^{j}, \\
\widetilde{q}^{-}(u, v) & =\sum_{i+j=0}^{2} \widetilde{b}_{i j}^{-} u^{i} v^{j} \\
& =\sum_{i+j=0}^{2} \frac{b_{i j}^{-}}{\left(a_{1}^{2}-4 a_{0} a_{2}\right)^{1 / 4}}\left[\frac{u}{\left(a_{1}^{2}-4 a_{0} a_{2}\right)^{1 / 4}}+\alpha_{S_{1}}\right]^{i} v^{j} .
\end{aligned}
$$

For $\varepsilon=0$, the Hamiltonian function of (18) has the form

$$
\widetilde{H}(u, v)=\frac{1}{2}\left(v^{2}-u^{2}\right)+\frac{a_{2}}{3\left(a_{1}^{2}-4 a_{0} a_{2}\right)^{3 / 4}} u^{3}+h_{1}
$$

and the corresponding first order Melinikov function can be denoted by $\widetilde{M}(h)$. By Remark 3.1.4 of [18], we can know that $M_{3}^{-}(h)=\widetilde{M}(h)$, and then, from Corollary 3.2.2 and the Theorem 3.2.1 of [18], $I_{1}$ can be written as

$$
\begin{aligned}
I_{1}(h)= & c_{1}\left(h-h_{1}\right) \ln \left(h_{1}-h\right)+c_{3}\left(h-h_{1}\right)^{2} \ln \left(h_{1}-h\right) \\
& +O\left(\left(h-h_{1}\right)^{2}\right), \quad 0<h_{1}-h \ll 1,
\end{aligned}
$$

where

$$
\begin{aligned}
c_{1}= & -\left(\widetilde{a}_{10}^{-}+\widetilde{b}_{01}^{-}\right)=-\frac{1}{\left(a_{1}^{2}-4 a_{0} a_{2}\right)^{1 / 4}} \\
& \times\left[\left(a_{10}^{-}+b_{01}^{-}\right)+\alpha_{S_{1}}\left(2 a_{20}^{-}+b_{11}^{-}\right)\right], \\
c_{3}= & \frac{3}{2} \times \frac{a_{2}}{3\left(a_{1}^{2}-4 a_{0} a_{2}\right)^{3 / 4}} \times \frac{1}{\left(a_{1}^{2}-4 a_{0} a_{2}\right)^{2 / 4}}\left(2 \widetilde{a}_{20}+\widetilde{b}_{11}\right) \\
= & \frac{a_{2}}{2\left(a_{1}^{2}-4 a_{0} a_{2}\right)^{5 / 4}}\left(2 a_{20}^{-}+b_{11}^{-}\right) .
\end{aligned}
$$

Thus, by the above discussion, one can obtain the expression of (13) and the formulas for $c_{1}$ and $c_{3}$. Now, we give the expressions of $c_{0}$ and $c_{2}$ in (13).

On the curve $L_{3 h}$ intersect $y$-axis with two points denoted by $A_{1}(h)=(0,-\sqrt{2 h})$ and $A(h)=(0, \sqrt{2 h})$. Then, from (12), we have

$$
\begin{aligned}
M_{3}^{-}(h) & =\int_{A_{1}(\overline{h) A}(h)} \sum_{i+j=0}^{2} b_{i j}^{-} x^{i} y^{j} d x-\sum_{i+j=0}^{2} a_{i j}^{-} x^{i} y^{j} d y \\
& =N_{1}(h)+N_{2}(h),
\end{aligned}
$$

where

$$
\begin{aligned}
N_{1}(h)= & \int_{A_{1} \overline{(h) A(h)}} \frac{}{\cup(h) A_{1}(h)} \sum_{i+j=0}^{2} b_{i j}^{-} x^{i} y^{j} d x \\
& -\sum_{i+j=0}^{2} a_{i j}^{-} x^{i} y^{j} d y, \\
N_{2}(h)= & \int_{\overline{A(h) A_{1}(h)}}\left(a_{00}^{-}+a_{01}^{-} y+a_{02}^{-} y^{2}\right) d y .
\end{aligned}
$$

By Green's formula two times to $N_{1}(h)$, we obtain

$$
\begin{aligned}
& N_{1}(h)=\int_{\text {Int. }\left(A_{1} \overline{(h) A}(h) \cup \overline{\left.A(h) A_{1}(h)\right)}\right.}\left[\left(a_{10}^{-}+b_{01}^{-}\right)+\left(b_{11}^{-}+2 a_{20}^{-}\right) x\right. \\
& \left.+\left(a_{11}^{-}+2 b_{02}^{-}\right) y\right] d x d y \\
& =\oint_{A_{1} \overline{(h) A(h)} \cup \overrightarrow{A(h) A_{1}(h)}}\left[\left(a_{10}^{-}+b_{01}^{-}\right) y+\left(b_{11}^{-}+2 a_{20}^{-}\right) x y\right. \\
& \left.+\frac{1}{2}\left(a_{11}^{-}+2 b_{02}^{-}\right) y^{2}\right] d x \\
& =\int_{L_{3 h}^{-}}\left[\left(a_{10}^{-}+b_{01}^{-}\right) y+\left(b_{11}^{-}+2 a_{20}^{-}\right) x y\right. \\
& \left.+\frac{1}{2}\left(a_{11}^{-}+2 b_{02}^{-}\right) y^{2}\right] d x .
\end{aligned}
$$

Further, one has

$$
\begin{aligned}
N_{2}(h) & =\int_{\overrightarrow{A(h) A_{1}(h)}}\left(a_{00}^{-}+a_{01}^{-} y+a_{02}^{-} y^{2}\right) d y \\
& =\int_{\sqrt{2 h}}^{-\sqrt{2 h}}\left(a_{00}^{-}+a_{01}^{-} y+a_{02}^{-} y^{2}\right) d y \\
& =-2 a_{00}^{-} \sqrt{2 h}-\frac{4}{3} h \sqrt{2 h} a_{02}^{-} .
\end{aligned}
$$

Thus, we have

$$
\begin{gathered}
M_{3}^{-}(h)=\int_{L_{3 h}^{-}}\left[\left(a_{10}^{-}+b_{01}^{-}\right) y+\left(b_{11}^{-}+2 a_{20}^{-}\right) x y\right. \\
\left.+\frac{1}{2}\left(a_{11}^{-}+2 b_{02}^{-}\right) y^{2}\right] d x \\
-2 a_{00}^{-} \sqrt{2 h}-\frac{4}{3} h \sqrt{2 h} a_{02}^{-}
\end{gathered}
$$


Then, let $h \rightarrow h_{1}$. Then, by (11), we can obtain the expression of $c_{0}$ in (13). Further, differentiating (13) with respect to $h$, together with Lemma 3.1.2 of [18], we obtain

$$
\begin{aligned}
\frac{d}{d h} M_{3}^{-}(h)= & \left.\int_{L_{3 h}^{-}}\left(p_{x}+q_{y}\right)\right|_{\varepsilon=0} d t \\
= & \int_{L_{3 h}^{-}}\left[\left(a_{10}^{-}+b_{01}^{-}\right)+\left(b_{11}^{-}+2 a_{20}^{-}\right) x\right. \\
& \left.+\left(a_{11}^{-}+2 b_{02}^{-}\right) y\right] d t-\frac{\sqrt{2 h}}{h} a_{00}^{-}-2 \sqrt{2 h} a_{02}^{-} \\
= & c_{1} \ln \left(h_{1}-h\right)+c_{1}+c_{2} \\
& +O\left(\left(h-h_{1}\right) \ln \left(h_{1}-h\right)\right),
\end{aligned}
$$

which, as $c_{1}=0$, gives that

$$
\begin{aligned}
\int_{L_{3 h}^{-}} & {\left[\left(a_{10}^{-}+b_{01}^{-}\right)+\left(b_{11}^{-}+2 a_{20}^{-}\right) x+\left(a_{11}^{-}+2 b_{02}^{-}\right) y\right] d t } \\
& -\frac{\sqrt{2 h}}{h} a_{00}^{-}-2 \sqrt{2 h} a_{02}^{-} \\
= & c_{2}+O\left(\left(h-h_{1}\right) \ln \left(h_{1}-h\right)\right) .
\end{aligned}
$$

Let $h \rightarrow h_{1}$ in the above. Then we can easily obtain the formula for $c_{2}$ in (13). This ends the proof.

In the same way, one can obtain the expressions of $M_{3}^{+}(h)$.

Lemma 3. For $M_{3}^{-}(h)$ in (12), one has

$$
\begin{array}{r}
M_{3}^{+}(h)=\widetilde{c}_{0}+\widetilde{c}_{1}\left(h-h_{1}\right) \ln \left(h_{1}-h\right)+\widetilde{c}_{2}\left(h-h_{1}\right) \\
+\widetilde{c}_{3}\left(h-h_{1}\right)^{2} \ln \left(h_{1}-h\right)+O\left(\left(h-h_{1}\right)^{2}\right), \\
0<h_{1}-h \ll 1,
\end{array}
$$

where

$$
\begin{gathered}
\widetilde{c}_{0}=\int_{L^{+}}\left[\left(a_{10}^{+}+b_{01}^{+}\right) y+\left(b_{11}^{+}+2 a_{20}^{+}\right) x y\right. \\
\left.\quad+\frac{1}{2}\left(a_{11}^{+}+2 b_{02}^{+}\right) y^{2}\right] d x+2 \sqrt{2 h_{1}} a_{00}^{+} \\
+\frac{4}{3} h_{1} \sqrt{2 h_{1}} a_{02}^{+}, \\
\widetilde{c}_{1}=-\frac{1}{\left(b_{1}^{2}-4 b_{0} b_{2}\right)^{1 / 4}}\left[\left(a_{10}^{+}+b_{01}^{+}\right)+\alpha_{S_{2}}\left(2 a_{20}^{+}+b_{11}^{+}\right)\right], \\
\left.\widetilde{c}_{2}\right|_{\widetilde{c}_{1}=0}=\int_{L^{+}}\left[\left(a_{10}^{+}+b_{01}^{+}\right)+\left(b_{11}^{+}+2 a_{20}^{+}\right) x+\left(a_{11}^{+}+2 b_{02}^{+}\right) y\right] d t \\
+\frac{\sqrt{2 h_{1}}}{h_{1}} a_{00}^{+}+2 \sqrt{2 h_{1}} a_{02}^{+}, \\
\widetilde{c}_{3}=\frac{b_{2}}{2\left(b_{1}^{2}-4 b_{0} b_{2}\right)^{5 / 4}}\left(2 a_{20}^{+}+b_{11}^{+}\right) .
\end{gathered}
$$

By applying Theorem 3.2.3 of [18], together with the proof of Lemma 2, we have the following.

Lemma 4. The functions $M_{i}(h), i=1,2$, given in (12) can be expressed as

$$
\begin{aligned}
M_{1}(h)= & c_{01}+c_{1}\left(h-h_{1}\right) \ln \left(h_{1}-h\right)+c_{21}\left(h-h_{1}\right) \\
& +c_{3}\left(h-h_{1}\right)^{2} \ln \left(h_{1}-h\right) \\
& +O\left(\left(h-h_{1}\right)^{2}\right), \quad 0<h_{1}-h \ll 1, \\
M_{2}(h)= & c_{02}+\widetilde{c}_{1}\left(h-h_{1}\right) \ln \left(h_{1}-h\right)+c_{22}\left(h-h_{1}\right) \\
& +\widetilde{c}_{3}\left(h-h_{1}\right)^{2} \ln \left(h_{1}-h\right) \\
& +O\left(\left(h-h_{1}\right)^{2}\right), \quad 0<h_{1}-h \ll 1,
\end{aligned}
$$

where $c_{1}, c_{3}, \widetilde{c}_{1}, \widetilde{c}_{3}$ are given in Lemmas 2 and 3 , and

$$
\begin{gathered}
c_{01}=\oint_{L_{1}} \sum_{i+j=0}^{2} b_{i j}^{-} x^{i} y^{j} d x-\sum_{i+j=0}^{2} a_{i j}^{-} x^{i} y^{j} d y, \\
c_{02}=\oint_{L_{2}} \sum_{i+j=0}^{2} b_{i j}^{+} x^{i} y^{j} d x-\sum_{i+j=0}^{2} a_{i j}^{+} x^{i} y^{j} d y, \\
\left.c_{21}\right|_{c_{1}=0}=\oint_{L_{1}}\left[\left(a_{10}^{-}+b_{01}^{-}\right)+\left(2 a_{20}^{-}+b_{11}^{-}\right) x\right. \\
\left.+\left(a_{11}^{-}+2 b_{02}^{-}\right) y\right]\left.d t\right|_{\mathcal{c}_{1}=0}, \\
\left.c_{22}\right|_{\widetilde{c}_{1}=0}=\oint_{L_{2}}\left[\left(a_{10}^{+}+b_{01}^{+}\right)+\left(2 a_{20}^{+}+b_{11}^{+}\right) x\right. \\
\left.+\left(a_{11}^{+}+2 b_{02}^{+}\right) y\right]\left.d t\right|_{\widetilde{c}_{1}=0} .
\end{gathered}
$$

By above three Lemmas 2, 3, and 4, together with (12), one can derive the following.

Lemma 5. For $M_{3}(h)$ and $M(h)$, one has

$$
\begin{aligned}
M_{3}(h)= & \left(c_{0}+\widetilde{c}_{0}\right)+\left(c_{1}+\widetilde{c}_{1}\right)\left(h-h_{1}\right) \ln \left(h_{1}-h\right) \\
& +\left(c_{2}+\widetilde{c}_{2}\right)\left(h-h_{1}\right)+\left(c_{3}+\widetilde{c}_{3}\right)\left(h-h_{1}\right)^{2} \ln \left(h_{1}-h\right) \\
& +\left(O\left(h_{1}-h\right)^{2}\right), \quad 0<h_{1}-h \ll 1, \\
M(h)= & \left(c_{0}+c_{01}+\widetilde{c}_{0}+c_{02}\right)+2\left(c_{1}+\widetilde{c}_{1}\right) \\
& \times\left(h-h_{1}\right) \ln \left(h-h_{1}\right)+\left(c_{2}+c_{21}+\widetilde{c}_{2}+c_{22}\right) \\
& \times\left(h-h_{1}\right)+2\left(c_{3}+\widetilde{c}_{3}\right)\left(h-h_{1}\right)^{2} \ln \left(h-h_{1}\right) \\
& +\left(O\left(h_{1}-h\right)^{2}\right), \quad 0<h-h_{1} \ll 1,
\end{aligned}
$$

where the corresponding coefficients are given in (14), (32), and (34).

Then, using Lemmas 4 and 5, we can have the following. 
Theorem 6. Let $(H)$ and (7) hold. Then if there exists $\delta_{0}$ such that

$$
\begin{gathered}
c_{01}\left(\delta_{0}\right)=c_{02}\left(\delta_{0}\right)=\left(c_{0}+\widetilde{c}_{0}\right)\left(\delta_{0}\right)=\left(c_{1}+\widetilde{c}_{1}\right)\left(\delta_{0}\right) \\
=\left(c_{2}+\widetilde{c}_{2}\right)\left(\delta_{0}\right)=0, \\
\left(c_{3}+\widetilde{c}_{3}\right)\left(\delta_{0}\right)<0, \quad c_{1}\left(\delta_{0}\right)<0, \quad \widetilde{c}_{1}\left(\delta_{0}\right)>0, \\
\left(c_{1}+\widetilde{c}_{1}\right)\left(\delta_{0}\right)=0, \quad\left(c_{21}+\widetilde{c}_{22}\right)\left(\delta_{0}\right)=0, \\
\operatorname{rank} \frac{\partial\left(c_{01}, c_{02}, c_{0}+\widetilde{c}_{0}, c_{1}+\widetilde{c}_{1}, c_{2}+\widetilde{c}_{2}\right)}{\partial \delta}=5,
\end{gathered}
$$

then system (1) can have 7 limit cycles near $L$.

Proof. By the assumption and from the expansions in Lemmas 4 and 5 we have

$$
\begin{aligned}
& M_{1}(h)=c_{1}\left(\delta_{0}\right)\left(h-h_{1}\right) \ln \left(h_{1}-h\right)+O\left(h_{1}-h\right)<0, \\
& 0<h_{1}-h \ll 1 \text {, } \\
& M_{2}(h)=\widetilde{c}_{1}\left(\delta_{0}\right)\left(h-h_{1}\right) \ln \left(h_{1}-h\right)+O\left(h-h_{1}\right)>0 \text {, } \\
& 0<h_{1}-h \ll 1 \text {, } \\
& M_{3}(h)=\left(c_{3}+\tilde{c}_{3}\right)\left(\delta_{0}\right)\left(h-h_{1}\right)^{2} \ln \left(h_{1}-h\right) \\
& +\left(O\left(h_{1}-h\right)^{2}\right)>0, \quad 0<h_{1}-h \ll 1, \\
& M(h)=2\left(c_{3}+\widetilde{c}_{3}\right)\left(h-h_{1}\right)^{2} \ln \left(h-h_{1}\right)+\left(O\left(h_{1}-h\right)^{2}\right)>0, \\
& 0<h-h_{1} \ll 1 .
\end{aligned}
$$

Note that $c_{01}, c_{02}, c_{0}+\widetilde{c}_{0}, c_{1}+\widetilde{c}_{1}$, and $c_{2}+\widetilde{c}_{2}$ can be taken as free parameters. Then we can vary them one by one to change the signs of $M_{1}, M_{2}, M_{3}$, and $M$.

First, let $c_{01}=c_{02}=c_{0}+\widetilde{c}_{0}=c_{1}+\widetilde{c}_{1}=0$ and $0<-\left(c_{2}+\widetilde{c}_{2}\right) \ll$ 1. Then, in this case, we have

$$
\begin{gathered}
M_{1}(h)<0, \quad M_{2}(h)>0, \quad M_{3}(h)>0, \\
\text { for } 0<h_{1}-h \ll 1, \\
M(h)<0, \quad \text { for } 0<h-h_{1} \ll 1,
\end{gathered}
$$

which means that there exists a zero $\widetilde{h}_{1}$ with $0<\widetilde{h}_{1}-h_{1}$ such that $M\left(\tilde{h}_{1}\right)=0$.

Second, fix $c_{01}=c_{02}=c_{0}+\widetilde{c}_{0}=0$ and vary $0 \ll-\left(c_{1}+\widetilde{c}_{1}\right) \ll$ $-\left(c_{2}+\widetilde{c}_{2}\right)$, so that

$$
\begin{gathered}
M_{1}(h)<0, \quad M_{2}(h)>0, \quad M_{3}(h)<0, \\
\text { for } 0<h_{1}-h \ll 1, \\
M(h)>0, \quad \text { for } 0<h-h_{1} \ll 1 .
\end{gathered}
$$

Hence, there exist $h_{1}-\tilde{h}_{2}>0, \tilde{h}_{3} \in\left(h_{1}, \tilde{h}_{1}\right)$ such that $M_{3}\left(\widetilde{h}_{2}\right)=0, M\left(\widetilde{h}_{3}\right)=0$.
Third, let $c_{01}=c_{0}+\widetilde{c}_{0}=0,0<-c_{02} \ll-\left(c_{1}+\widetilde{c}_{1}\right)$, implying

$$
\begin{gathered}
M_{1}(h)<0, \quad M_{2}(h)<0, \quad M_{3}(h)<0, \\
\text { for } 0<h_{1}-h \ll 1, \\
M(h)<0, \quad \text { for } 0<h-h_{1} \ll 1 .
\end{gathered}
$$

Then $M_{2}\left(\widetilde{h}_{4}\right)=0, M\left(\widetilde{h}_{5}\right)=0$ for some $\widetilde{h}_{4}<h_{1}, \widetilde{h}_{5} \in\left(h_{1}, \widetilde{h}_{3}\right)$.

Fourth, let $c_{0}+\widetilde{c}_{0}=0$ and $0<c_{01} \ll-c_{02}$; we have

$$
\begin{gathered}
M_{1}(h)>0, \quad M_{2}(h)<0, \quad M_{3}(h)<0, \\
\text { for } 0<h_{1}-h \ll 1, \\
M(h)<0, \quad \text { for } 0<h-h_{1} \ll 1 .
\end{gathered}
$$

So there exists $\widetilde{h}_{6}<h_{1}$ such that $M_{1}\left(\widetilde{h}_{6}\right)=0$.

Finally, vary $0<c_{0}+\widetilde{c}_{0} \ll c_{01}$. In this case,

$$
\begin{gathered}
M_{1}(h)>0, \quad M_{2}(h)<0, \quad M_{3}(h)>0, \\
\text { for } 0<h_{1}-h \ll 1, \\
M(h)<0, \quad \text { for } 0<h-h_{1} \ll 1 .
\end{gathered}
$$

So there exists $\widetilde{h}_{7} \in\left(\widetilde{h}_{2}, h_{1}\right)$ such that $M_{3}\left(\widetilde{h}_{7}\right)=0$.

In a word, we have proved that if

$$
0<c_{0}+\widetilde{c}_{0} \ll c_{01} \ll-c_{02} \ll-\left(c_{1}+\widetilde{c}_{1}\right) \ll-\left(c_{2}+\widetilde{c}_{2}\right) \ll 1
$$

then $M_{1}, M_{2}, M_{3}$, and $M$ have one zero, one zero, two zeros, and three zeros which implies that seven limit cycles can appear in the neighbourhood of $L$.

2.2. Proofs. In this section, we will use Theorem 6 to prove our main result. For that purpose, let

$$
\begin{array}{lll}
a_{2}=-1, & a_{1}=-\frac{3}{2}, & a_{0}=-\frac{1}{2}, \\
b_{2}=1, & b_{1}=-\frac{11}{3}, & b_{0}=\frac{41}{48} .
\end{array}
$$

Then, the Hamiltonian functions in (5) can be rewritten as

$$
\begin{aligned}
& H^{-}(x, y)=\frac{1}{2} y^{2}-\frac{1}{3} x^{3}-\frac{3}{4} x^{2}-\frac{1}{2} x, \\
& H^{+}(x, y)=\frac{1}{2} y^{2}+\frac{1}{3} x^{3}-\frac{11}{6} x^{2}+\frac{11}{48} x .
\end{aligned}
$$

And in this case

$$
\begin{gathered}
S_{1}=\left(-\frac{1}{2}, 0\right), \quad S_{2}=\left(\frac{1}{4}, 0\right), \quad C_{1}=(-1,0), \\
C_{2}=\left(\frac{41}{12}, 0\right), \quad h_{1}=\frac{5}{48} .
\end{gathered}
$$

Under (44), it is not hard to obtain the expressions for $c_{1}$, $\widetilde{c}_{1}, c_{3}$, and $\widetilde{c}_{3}$ in Lemmas 2 and 3 . 
Lemma 7. Under (44), for system (1), one has

$$
\begin{aligned}
& c_{1}=-\sqrt{2}\left[\left(a_{10}^{-}+b_{01}^{-}\right)-\frac{1}{2}\left(2 a_{20}^{-}+b_{11}^{-}\right)\right], \\
& c_{3}=-\sqrt{2}\left(2 a_{20}^{-}+b_{11}^{-}\right), \\
& \widetilde{c}_{1}=-\sqrt{\frac{6}{19}}\left[\left(a_{10}^{+}+b_{01}^{+}\right)+\frac{1}{4}\left(2 a_{20}^{+}+b_{11}^{+}\right)\right], \\
& \widetilde{c}_{3}=\frac{1}{2}\left(\frac{6}{19}\right)^{5 / 2}\left(2 a_{20}^{+}+b_{11}^{+}\right) .
\end{aligned}
$$

For the other expressions of coefficients in Lemmas 4 and 5 , one has the following.

Lemma 8. Under (44), for system (1), one has

$$
\begin{aligned}
& c_{01}=\frac{3 \sqrt{2}}{20}\left(a_{10}^{-}+b_{01}^{-}\right)-\frac{39 \sqrt{2}}{280}\left(2 a_{20}^{-}+b_{11}^{-}\right), \\
& c_{21}=-3 \sqrt{2}\left(2 a_{20}^{-}+b_{11}^{-}\right), \\
& c_{02}=\frac{361 \sqrt{114}}{180}\left(a_{10}^{+}+b_{01}^{+}\right)+\frac{29963 \sqrt{114}}{5040}\left(2 a_{20}^{+}+b_{11}^{+}\right) \text {, } \\
& c_{22}=\sqrt{114}\left(2 a_{20}^{+}+b_{11}^{+}\right) \text {, } \\
& c_{0}=\frac{3 \sqrt{2}}{20}\left(a_{10}^{-}+b_{01}^{-}\right)-\left(\frac{39 \sqrt{2}}{280}-\frac{5 \sqrt{30}}{168}\right)\left(2 a_{20}^{-}+b_{11}^{-}\right) \\
& -\frac{\sqrt{30}}{6} a_{00}^{-}-\frac{5 \sqrt{30}}{432} a_{02}^{-} \\
& \widetilde{c}_{0}=\left(-\frac{35}{9} \sqrt{30}+\frac{361}{180} \sqrt{114}\right)\left(a_{10}^{+}+b_{01}^{+}\right) \\
& +\left(\frac{29963}{5040} \sqrt{114}-\frac{730}{63} \sqrt{30}\right)\left(2 a_{20}^{+}+b_{11}^{+}\right)+\frac{\sqrt{30}}{6} a_{00}^{+} \\
& +\frac{5 \sqrt{30}}{432} a_{02}^{+} \\
& c_{2}=(\sqrt{30}-3 \sqrt{2})\left(2 a_{20}^{-}+b_{11}^{-}\right)-\frac{4 \sqrt{30}}{5} a_{00}^{-}-\frac{\sqrt{30}}{6} a_{02}^{-} \text {, } \\
& \widetilde{c}_{2}=(-2 \sqrt{30}+\sqrt{114})\left(2 a_{20}^{+}+b_{11}^{+}\right)+\frac{4 \sqrt{30}}{5} a_{00}^{+}+\frac{\sqrt{30}}{6} a_{02}^{+} \text {. }
\end{aligned}
$$

Proof. Note that $c_{01}=\oint_{L_{1}} \sum_{i+j=0}^{2} b_{i j}^{-} x^{i} y^{j} d x-\sum_{i+j=0}^{2} a_{i j}^{-} x^{i} y^{j} d y$.

Then, by Green's formula two times, we derive

$$
\begin{gathered}
c_{01}=\int_{\text {Int. }\left(L_{1}\right)}\left[\left(a_{10}^{-}+b_{01}^{-}\right)+\left(b_{11}^{-}+2 a_{20}^{-}\right) x\right. \\
\left.+\left(a_{11}^{-}+2 b_{02}^{-}\right) y\right] d x d y \\
=\oint_{L_{1}}\left[\left(a_{10}^{-}+b_{01}^{-}\right) y+\left(b_{11}^{-}+2 a_{20}^{-}\right) x y\right. \\
\left.+\frac{1}{2}\left(a_{11}^{-}+2 b_{02}^{-}\right) y^{2}\right] d x .
\end{gathered}
$$

On the other hand, along the curve $L_{1}$, we have $(1 / 2) y^{2}-$ $(1 / 3) x^{3}-(3 / 4) x^{2}-(1 / 2) x=5 / 48$, for $-(5 / 4) \leq x \leq-(1 / 2)$. Then, (49) can be represented as

$$
\begin{aligned}
c_{01}=2 \int_{-5 / 4}^{-1 / 2} & {\left[\left(a_{10}^{-}+b_{01}^{-}\right)+\left(b_{11}^{-}+2 a_{20}^{-}\right) x\right] } \\
& \times \sqrt{\frac{5}{24}+\frac{2}{3} x^{3}+\frac{3}{2} x^{2}+x} d x .
\end{aligned}
$$

By applying Maple, one can compute that

$$
\begin{gathered}
\int_{-5 / 4}^{-1 / 2} \sqrt{\frac{5}{24}+\frac{2}{3} x^{3}+\frac{3}{2} x^{2}+x} d x=\frac{3 \sqrt{2}}{40} \\
\int_{-5 / 4}^{-1 / 2} x \sqrt{\frac{5}{24}+\frac{2}{3} x^{3}+\frac{3}{2} x^{2}+x} d x=-\frac{39 \sqrt{2}}{560} .
\end{gathered}
$$

Inserting the above into (50) gives the expression of $c_{01}$.

Recall that $c_{1}=0$ if and only if $a_{10}^{-}+b_{01}^{-}=(1 / 2)\left(2 a_{20}^{-}+b_{11}^{-}\right)$. Thus, by $\left.(4 \mathrm{a})\right|_{\varepsilon=0}$, we rewrite $\left.c_{21}\right|_{c_{1}=0}$ as

$$
\begin{aligned}
& \left.c_{21}\right|_{c_{1}=0} \\
& =\oint_{L_{1}} \frac{\left[(1 / 2)\left(2 a_{20}^{-}+b_{11}^{-}\right)+\left(2 a_{20}^{-}+b_{11}^{-}\right) x+\left(a_{11}^{-}+2 b_{02}^{-}\right) y\right]}{y} d x \\
& =\frac{1}{2}\left(2 a_{20}^{-}+b_{11}^{-}\right) \oint_{L_{1}} \frac{1+2 x}{y} d x \\
& =\left(2 a_{20}^{-}+b_{11}^{-}\right) \int_{-5 / 4}^{-1 / 2} \frac{1+2 x}{\sqrt{(5 / 24)+(2 / 3) x^{3}+(3 / 2) x^{2}+x}} d x,
\end{aligned}
$$

which, by Maple, yields the conclusion for $c_{02}$. Using a similar way, one can derive the formulas for $c_{02}$ and $c_{22}$. Now, we deduce the formulas for $c_{0}$ and $c_{2}$.

Note that, on the curve $L^{-}:(1 / 2) y^{2}-(1 / 3) x^{3}-(3 / 4) x^{2}-$ $(1 / 2) x=(5 / 48),-(1 / 2)<x<0$, we have

$$
\begin{aligned}
c_{0}=2 \int_{-1 / 2}^{0} & {\left[\left(a_{10}^{-}+b_{01}^{-}\right)+\left(b_{11}^{-}+2 a_{20}^{-}\right) x\right] } \\
& \times \sqrt{\frac{5}{24}+\frac{2}{3} x^{3}+\frac{3}{2} x^{2}+x d x} \\
& -\frac{\sqrt{30}}{6} a_{00}^{-}-\frac{5 \sqrt{30}}{432} a_{02}^{-} .
\end{aligned}
$$

By Maple again, we obtain that

$$
\begin{gathered}
\int_{-1 / 2}^{0} \sqrt{\frac{5}{24}+\frac{2}{3} x^{3}+\frac{3}{2} x^{2}+x} d x=\frac{3 \sqrt{2}}{40} \\
\int_{-1 / 2}^{0} x \sqrt{\frac{5}{24}+\frac{2}{3} x^{3}+\frac{3}{2} x^{2}+x} d x=-\frac{39 \sqrt{2}}{560}+\frac{5 \sqrt{30}}{336} .
\end{gathered}
$$

Thus, we can obtain the conclusion for $c_{0}$. 
When $c_{1}=0$, we have $\left(a_{10}^{-}+b_{01}^{-}\right)=(1 / 2)\left(2 a_{20}^{-}+b_{11}^{-}\right)$. Thus, $c_{2}$ in (14) can be rewritten as

$$
\begin{aligned}
c_{2}= & \frac{1}{2}\left(2 a_{20}^{-}+b_{11}^{-}\right) \int_{L^{-}} \frac{1+2 x}{y} d x-\frac{2 a_{00}^{-}}{\sqrt{2 h_{1}}} \\
& -\left.\frac{4 a_{02}^{-}}{3}\left(\sqrt{2 h_{1}}+\frac{h_{1}}{\sqrt{2 h_{1}}}\right)\right|_{h_{1}=5 / 48} \\
= & \left(2 a_{20}^{-}+b_{11}^{-}\right) \int_{-1 / 2}^{0} \frac{1+2 x}{\sqrt{(5 / 24)+(2 / 3) x^{3}+(3 / 2) x^{2}+x}} d x \\
& -\frac{4}{5} \sqrt{6} a_{00}^{-}-\frac{\sqrt{30}}{6} a_{02}^{-},
\end{aligned}
$$

which, by Maple, implies the conclusion. Further, we can prove $\widetilde{c}_{0}$ and $\widetilde{c}_{2}$ in the same way to $c_{0}$ and $c_{2}$, respectively. This finishes the proof.

\section{Proof of Theorem 1. Let}

$$
\begin{array}{lll}
\delta_{1}=a_{10}^{-}+b_{01}^{-}, & \delta_{2}=2 a_{20}^{-}+b_{11}^{-}, & \delta_{3}=a_{10}^{+}+b_{01}^{+}, \\
\delta_{4}=2 a_{20}^{+}+b_{11}^{+}, & \delta_{5}=a_{00}^{+}-a_{00}^{-}, & \delta_{6}=a_{02}^{+}-a_{02}^{-} .
\end{array}
$$

Then, solve the equations

$$
c_{01}=c_{02}=c_{0}+\widetilde{c}_{0}=c_{1}+\widetilde{c}_{1}=c_{2}+\widetilde{c}_{2}=0,
$$

which, together with (56), gives that

$$
\begin{aligned}
& \delta_{1}=\frac{13 \sqrt{57}}{42} \delta_{4}, \quad \delta_{2}=\frac{\sqrt{57}}{3} \delta_{4}, \quad \delta_{3}=-\frac{83}{28} \delta_{4}, \\
& \delta_{5}=\left(\frac{5 \sqrt{57}}{42}-\frac{5}{7}\right) \delta_{4}, \quad \delta_{6}=\left(-\frac{18 \sqrt{57}}{7}+\frac{108}{7}\right) \delta_{4},
\end{aligned}
$$

where $\delta_{4}>0$. Then, under (58)

$$
\begin{gathered}
c_{1}=-\frac{\sqrt{114}}{7} \delta_{4}<0, \quad \widetilde{c}_{1}=\frac{\sqrt{114}}{7} \delta_{4}>0, \\
c_{3}+\widetilde{c}_{3}=-\frac{6805}{20577} \sqrt{114} \delta_{4}<0, \quad c_{1}+\widetilde{c}_{1}=0, \\
c_{21}+\widetilde{c}_{22}=0 .
\end{gathered}
$$

Further,

$$
\operatorname{rank} \frac{\partial\left(c_{01}, c_{02}, c_{0}+\widetilde{c}_{0}, c_{1}+\widetilde{c}_{1}, c_{2}+\widetilde{c}_{2}\right)}{\partial\left(\delta_{1}, \delta_{2}, \delta_{3}, \delta_{5}, \delta_{6}\right)}=5
$$

Thus, by Theorem 6 , there exist 7 limit cycles near $L$ for $\varepsilon>0$ small.
Under (58), we have for $0<(5 / 48)-h \ll 1$

$$
\begin{aligned}
M_{1}(h)= & -\frac{\sqrt{114}}{7} \delta_{4}\left(h-\frac{5}{48}\right) \ln \left(\frac{5}{48}-h\right) \\
& +O\left(\left(h-\frac{5}{48}\right)\right)<0, \\
M_{2}(h)= & \frac{\sqrt{114}}{7} \delta_{4}\left(h-\frac{5}{48}\right) \ln \left(\frac{5}{48}-h\right) \\
& +O\left(\left(h-\frac{5}{48}\right)\right)>0 .
\end{aligned}
$$

Now, we derive the expansion of $M_{1}(h)$. To achieve this, make the transformation

$$
u=\frac{\sqrt{2}}{2}(x+1), \quad v=y,
$$

together with time scaling $\tau=(\sqrt{2} / 2) t$. Then the system becomes

$$
\begin{aligned}
& \dot{u}=v+\varepsilon \bar{p}(u, v), \\
& \dot{v}=-u-2 \sqrt{2} u^{2}+\varepsilon \bar{q}^{-}(u, v),
\end{aligned}
$$

where

$$
\begin{aligned}
& \bar{p}(u, v)=\sum_{i+j=0}^{2} \bar{a}_{i j}^{-} u^{i} v^{j}=\sum_{i+j=0}^{2} a_{i j}^{-}(\sqrt{2} u-1)^{i} v^{j}, \\
& \bar{q}^{-}(u, v)=\sum_{i+j=0}^{2} \bar{b}_{i j}^{-} u^{i} v^{j}=\sum_{i+j=0}^{2} \sqrt{2} b_{i j}^{-}(\sqrt{2} u-1)^{i} v^{j} .
\end{aligned}
$$

For $\varepsilon=0$, the Hamiltonian function of (63) has the form

$$
\bar{H}(u, v)=\frac{1}{2}\left(v^{2}+u^{2}\right)+\frac{2 \sqrt{2}}{3} u^{3}+\frac{1}{12} .
$$

Denote $\bar{M}_{1}(h)$ by the first Melnikov function of system (63) near $C_{1}$, and note that

$$
q^{-} d x-p^{-} d y=\left(\frac{\sqrt{2}}{2} \bar{q}^{-}\right) \frac{d u}{(\sqrt{2} / 2)}-\bar{p}^{-} d v=\bar{q}^{-} d u-\bar{p}^{-} d v .
$$

Then we have $\bar{M}_{1}(h)=M_{1}(h)$. From Theorem 2.4.1 of [18], we can obtain

$$
\begin{aligned}
M_{1}(h)=\widetilde{b}_{0}\left(h-\frac{1}{12}\right)+O & \left(\left(h-\frac{1}{12}\right)^{2}\right)<0, \\
0 & <h-\frac{1}{12} \ll 1,
\end{aligned}
$$

where, under (58),

$$
\begin{aligned}
\widetilde{b}_{0} & =2 \pi\left(\bar{p}_{u}+\bar{q}_{v}^{-}\right)(0,0) \\
& =2 \sqrt{2} \pi\left[\left(a_{10}^{-}+b_{01}^{-}\right)-\left(2 a_{20}^{-}+b_{11}^{-}\right)\right]=2 \sqrt{2} \pi\left(\delta_{1}-\delta_{2}\right) \\
& =-\frac{\sqrt{114}}{21} \pi \delta_{4} .
\end{aligned}
$$


Similarly, under (58) we have

$$
\begin{array}{r}
M_{2}(h) \\
=-\frac{\sqrt{114}}{228} \delta_{4}\left(h+\frac{1681}{324}\right)+O\left(\left(h-h+\frac{1681}{324}\right)^{2}\right)<0, \\
0<h+\frac{1681}{324} \ll 1 .
\end{array}
$$

This implies that there exists another zero between $h=$ $-(1681 / 324)$ and $h=5 / 48$ for $M_{2}(h)$. This ends the proof.

\section{Conflict of Interests}

The author declares that there is no conflict of interests regarding the publication of this paper.

\section{Acknowledgments}

The author would like to give thanks to Professor Maoan Han for his helpful discussions during the preparation of the paper. The project was supported by National Natural Science Foundation of China (11271261), FP7-PEOPLE-2012-IRSES316338, and Shanghai Municipal Natural Science Foundation no. 12ZR1421600.

\section{References}

[1] M. di Bernardo, C. J. Budd, and A. R. Champneys, PiecewiseSmooth Dynamical Systems: Theory and Applications, vol. 163 of Applied Mathematical Sciences, Springer, London, UK, 2008.

[2] C. J. Budd, "Non-smooth dynamical systems and the grazing bifurcation," in Nonlinear Mathematics and Its Applications (Guildford, 1995), pp. 219-235, Cambridge University Press, Cambridge, UK, 1996.

[3] C. A. Buzzi, P. R. da Silva, and M. A. Teixeira, "A singular approach to discontinuous vector fields on the plane," Journal of Differential Equations, vol. 231, no. 2, pp. 633-655, 2006.

[4] J. Llibre, E. Ponce, and F. Torres, "On the existence and uniqueness of limit cycles in Liénard differential equations allowing discontinuities," Nonlinearity, vol. 21, no. 9, pp. 21212142, 2008.

[5] M. Han and W. Zhang, "On Hopf bifurcation in non-smooth planar systems," Journal of Differential Equations, vol. 248, no. 9, pp. C2399-C2416, 2010.

[6] F. Giannakopoulos and K. Pliete, "Planar systems of piecewise linear differential equations with a line of discontinuity," Nonlinearity, vol. 14, no. 6, pp. 1611-1632, 2001.

[7] F. Giannakopoulos and K. Pliete, "Closed trajectories in planar relay feedback systems," Dynamical Systems, vol. 17, no. 4, pp. 343-358, 2002.

[8] J. C. Artés, J. Llibre, J. C. Medrado, and M. A. Teixeira, "Piecewise linear differential systems with two real saddles," Mathematics and Computers in Simulation, vol. 95, pp. 13-22, 2014.

[9] J. Llibre, M. A. Teixeira, and J. Torregrosa, "Lower bounds for the maximum number of limit cycles of discontinuous piecewise linear differential systems with a straight line of separation," International Journal of Bifurcation and Chaos, vol. 23, no. 4, Article ID 1350066, 10 pages, 2013.

[10] J. Llibre and E. Ponce, "Three nested limit cycles in discontinuous piecewise linear differential systems with two zones," Dynamics of Continuous, Discrete and Impulsive Systems B, vol. 19, no. 3, pp. 325-335, 2012.

[11] S. Huan and X. Yang, "On the number of limit cycles in general planar piecewise linear systems of node-node types," Journal of Mathematical Analysis and Applications, vol. 411, no. 1, pp. 340353, 2014.

[12] F. Liang, M. Han, and V. G. Romanovski, "Bifurcation of limit cycles by perturbing a piecewise linear Hamiltonian system with a homoclinic loop," Nonlinear Analysis: Theory, Methods \& Applications, vol. 75, no. 11, pp. 4355-4374, 2012.

[13] Y. Xiong and M. Han, "Bifurcation of limit cycles by perturbing a piecewise linear Hamiltonian system," Abstract and Applied Analysis, vol. 2013, Article ID 575390, 19 pages, 2013.

[14] Y. Liu and Y. Xiong, "Bifurcation of limit cycles by perturbing a piecewise linear Hamiltonian system with one or two saddles," Chaos, Solitons \& Fractals, vol. 66, pp. 86-95, 2014.

[15] J. Llibre and A. C. Mereu, "Limit cycles for discontinuous quadratic differential systems with two zones," Journal of Mathematical Analysis and Applications, vol. 413, no. 2, pp. 763-775, 2014.

[16] Y. Xiong and M. Han, "Limit cycle bifurcations in a class of perturbed piecewise smooth systems," Applied Mathematics and Computation, vol. 242, pp. 47-64, 2014.

[17] X. Liu and M. Han, "Bifurcation of limit cycles by perturbing piecewise Hamiltonian systems," International Journal of Bifurcation and Chaos in Applied Sciences and Engineering, vol. 20, no. 5, pp. 1379-1390, 2010.

[18] M. Han, Bifurcation Theory of Limit Cycles, Science Press, Beijing, China, 2013. 


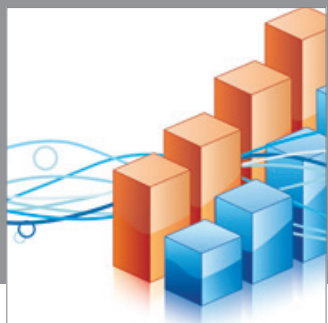

Advances in

Operations Research

mansans

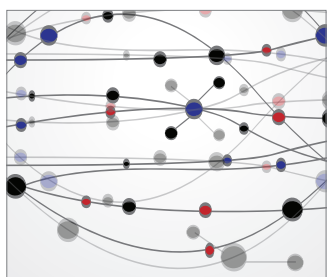

The Scientific World Journal
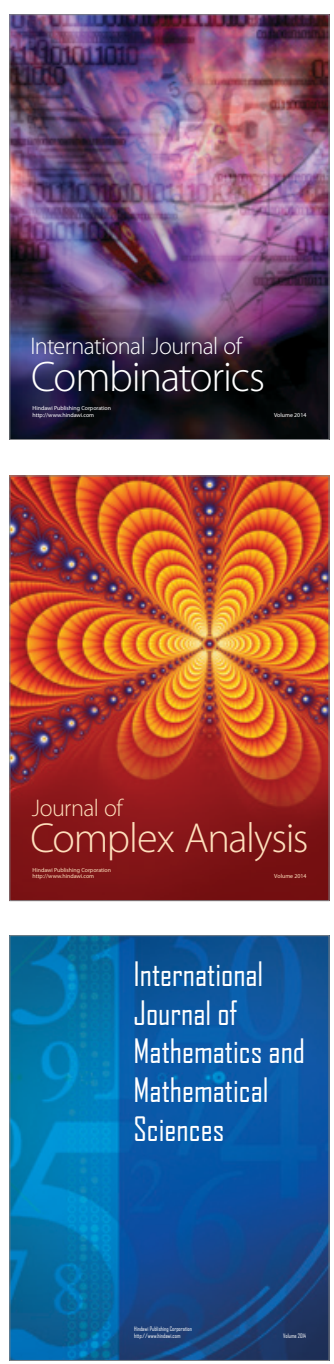
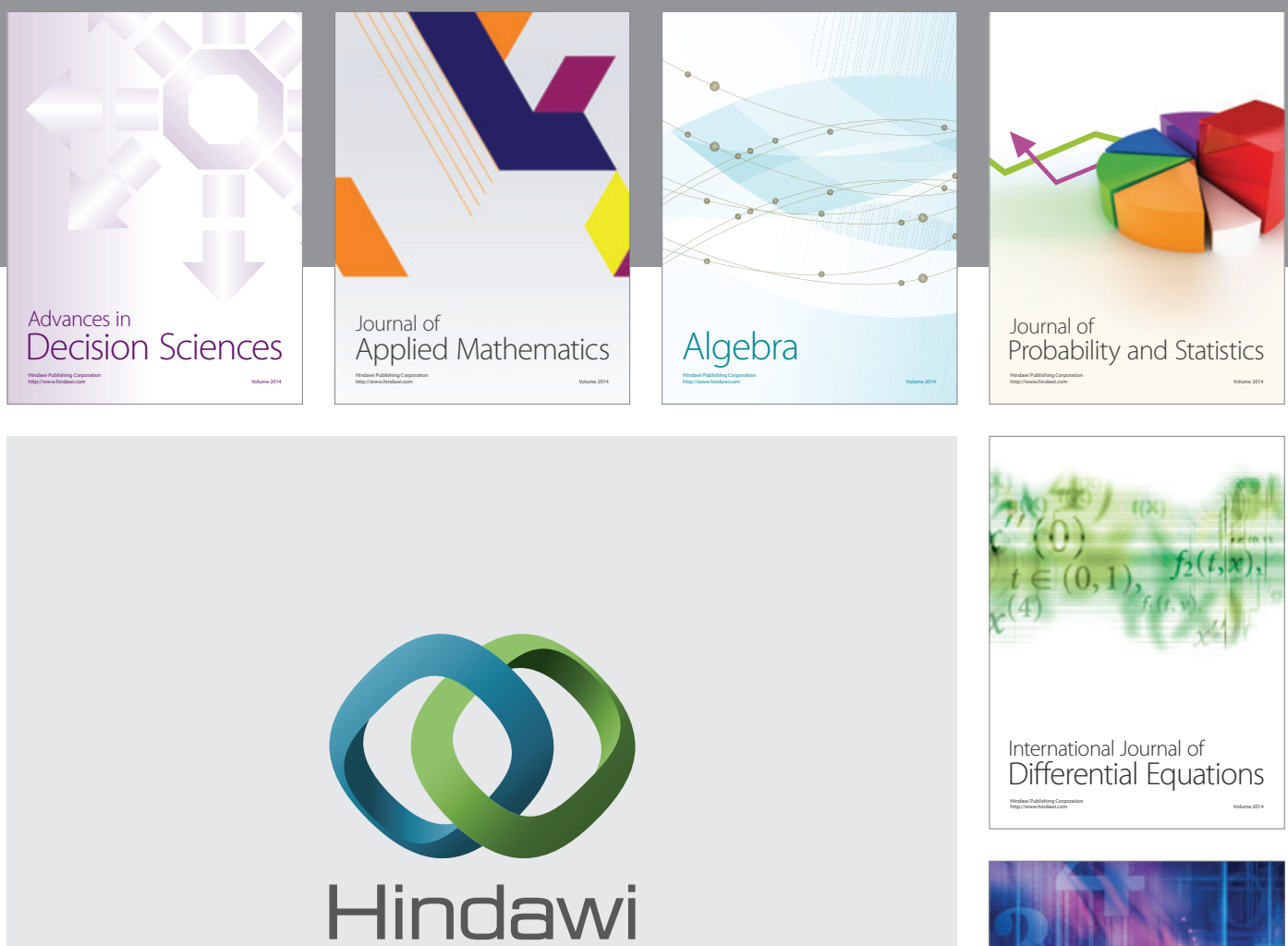

Submit your manuscripts at http://www.hindawi.com
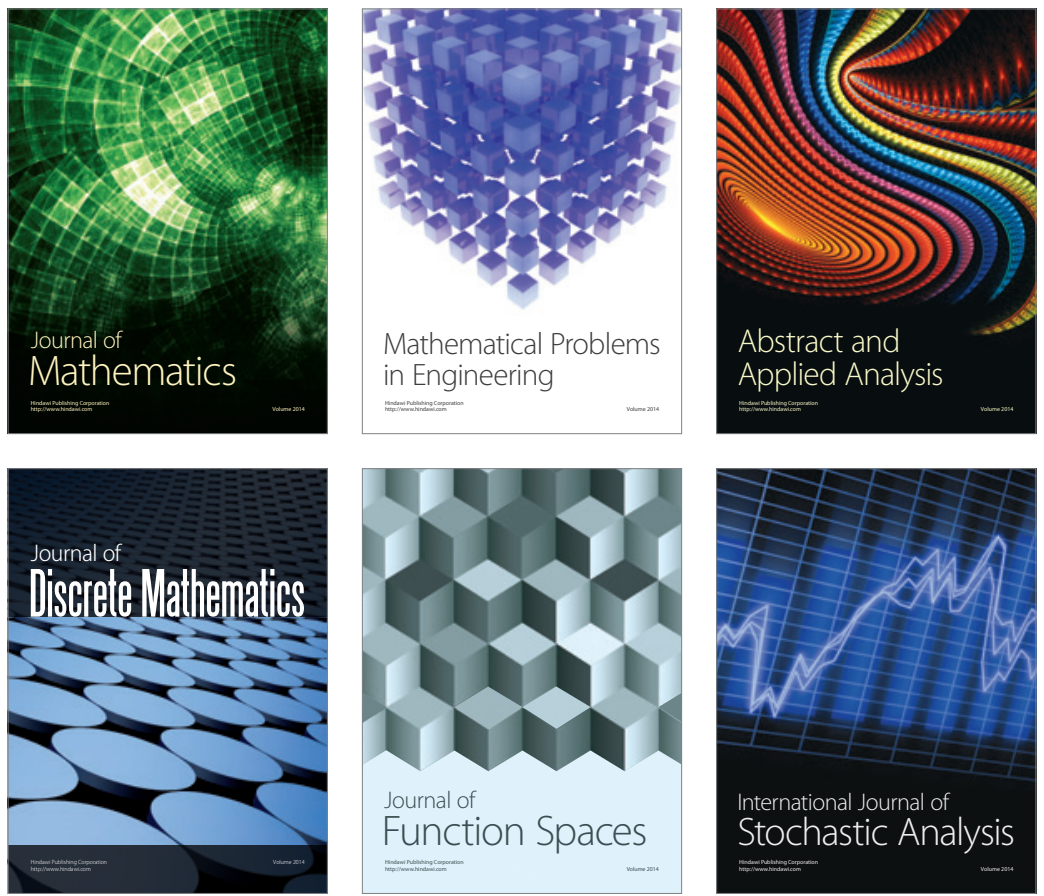

Journal of

Function Spaces

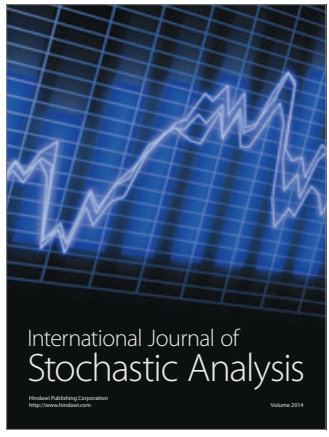

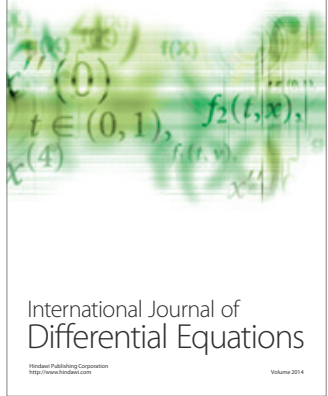
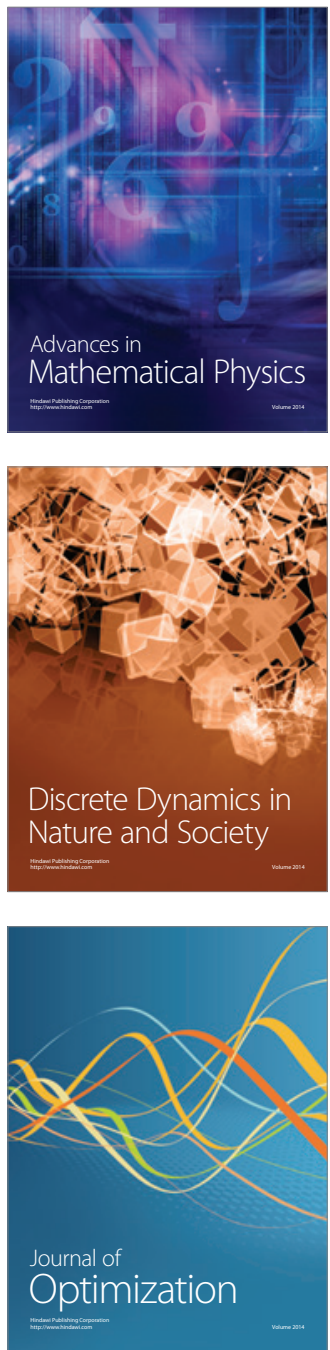